\title{
Identification of Bacterial Pathogens and their Antibiogram from Ascitic Fluid in a Rural Tertiary Care Hospital of North India
}

\author{
Kamlesh Thakur, Anuradha Sood, Subhash Chand Jaryal, \\ Puneet Kumar Gupta, Smriti Chauhan \\ Department of Microbiology, \\ Dr. Rajendra Prasad Government Medical College, Tanda, Himachal Pradesh.
}

\begin{abstract}
Background \& Objectives: The familiarity on the part of the clinician with ascitic fluid interpretation and with ascitic fluid characteristics in various diseases will increase the chances of controlling ascites early. The definite diagnosis of spontaneous bacterial peritonitis (SBP) is based on clinical evidence, neutrophil count and positive ascitic fluid culture. The objective of this study was to identify the bacterial agents from ascitic fluid and to determine their antibiotic resistance profile.
\end{abstract}

Material and Methods: A retrospective analysis of 132 ascitic fluid specimens collected for direct Gram's staining and culture during March 2012 to April 2013 was performed. The antibiotic susceptibility testing was done as per Clinical laboratory Standard Institute guidelines. The extended-spectrum beta-lactamase (ESBL) production in Gram negative isolates were determined.

Results : On Gram's staining, microorganisms with pus cells were seen in $20.4 \%$ and culture was positive in $37.8 \%$ of samples. Most of the isolates were Gram negative (70\%). Among Gram negative isolates, E. coli was most common isolate (74\%) followed by Acinetobacter spp. (11.6\%), Enterobacter spp. (5.8\%), Pseudomonas aeruginosa (5.8\%) and Klebsiella oxytoca (2.9\%). Among Gram positive isolates, Staphylococcus aureus, Enterococcus faecalis and coagulase negative staphylococci were $73.3 \%, 20 \%$ and $6.7 \%$ respectively. ESBLs were detected among $17.6 \%$ of $E$. coli and $30.5 \%$ of Klebsiella oxytoca isolates and all were multidrug resistant. All the strains were found to be sensitive to imipenem.

Interpretation \& Conclusion: The knowledge of presumptive causative organism and their antibiotic resistance profile will help the clinician in choosing empirical antibiotic therapy in suspected patients of SBP.

Keywords: Ascitic fluid, culture, sensitivity, spontaneous bacterial peritonitis.

\section{INTRODUCTION}

Diagnostic paracentesis with ascitic fluid analysis is crucial for the accurate diagnosis and management of ascites. Ascites is associated with several conditions

\section{Corresponding Author :}

Dr Puneet Kumar Gupta

Senior Resident,

Department of Microbiology,

Dr. Rajendra Prasad Government Medical College (DRPGMC),

Kangra at Tanda, Himachal Pradesh. PIN 176001

Email: drpuneetkumargupta@gmail.com

Mobile No: 088948-67079

Fax No: 01892-267115 such as chronic liver disease, congestive heart failure, nephrotic syndrome, tuberculosis (TB) and malignancy. Conn and Fessel ${ }^{1}$ described a syndrome of infected ascitic fluid in patients with hepatic cirrhosis and named as spontaneous bacterial peritonitis (SBP). SBP is defined as an infection of previously sterile ascitic fluid, without any apparent intra-abdominal source of infection. $^{2}$

The infecting organisms are usually those found among the normal intestinal flora. The criteria for diagnosis of SBP is the presence of $\geq 500 / \mathrm{mm}^{3}$ of leukocytes or presence of $\geq 250$ neutrophils $/ \mathrm{mm}^{3}$ in ascitic fluid with positive or negative culture. It may develop in individuals shown to have bacteria in their ascitic fluid 
prior to the development of an elevated polymorph nuclear (PMN) cell count or other evidence of local or systemic infection. There has been a condition resembling SBP that is characterized by PMN count of $<250$ cells $/ \mathrm{mm}^{3}$ with a negative culture known as culture negative neutrocytic ascites (CNNA). ${ }^{3}$ Familiarity on the part of the clinician with ascitic fluid interpretation and with ascitic fluid characteristics in various diseases will increase the chances of controlling ascites early. This study was planned with an objective to identify the bacterial isolates from ascitic fluid and to determine their antibiotic resistance pattern.

\section{MATERIAL AND METHODS}

A retrospective analysis of 132 ascitic fluid samples received in the Department of Microbiology, at a rural tertiary care center of North India was done from March 2012 to April 2013. Ten $\mathrm{ml}$ of sample received was divided into two portions, one was used for Gram's staining and another for the culture using blood culture bottle method as described by Siersema et al ${ }^{4}$ with slight modifications. Five $\mathrm{ml}$ of sample was centrifuged at $3000 \mathrm{~g}$ for 5 minutes and the deposit was used for Gram's staining. Another $5 \mathrm{ml}$ of sample was inoculated into 45 $\mathrm{ml}$ of brain heart infusion broth (Himedia Laboratories Pvt. Ltd. Mumbai) for enrichment and incubated aerobically at $37^{\circ} \mathrm{C}$. After 24 hours of incubation broth was sub- cultured on blood agar, MacConkey agar and chocolate agar. The isolates were identified using standard microbiological techniques. The antibiotic susceptibility testing of the culture isolates was performed for amoxicillin clavulanic acid combination (amoxyclav) $(20 / 10 \mu \mathrm{g})$, ceftazidime $(30 \mu \mathrm{g})$, cefotaxime $(30 \mu \mathrm{g})$, ceftriaxone $(30 \mu \mathrm{g})$, cefoperazone $(30 \mu \mathrm{g})$, amikacin $(30 \mu \mathrm{g})$, ciprofloxacin $(5 \mu \mathrm{g})$, imipenem $(10 \mu \mathrm{g})$, cefoxitin $(30 \mu \mathrm{g})$, clindamycin (10ug) and vancomycin $(10 \mu \mathrm{g})$ (Hi Media Pvt. Ltd Mumbai India) by Kirby Bauer's disc diffusion technique as per Clinical laboratory Standard Institute (CLSI) guidelines. ${ }^{5}$ The extended-spectrum beta-lactamase (ESBL) production in Gram negative isolates were determined using modified double disk synergy tests. ${ }^{6}$ Escherichia coli ATCC 25922 was used as a quality control strain.

\section{RESULTS}

The Gram's staining of ascitic fluid samples revealed microorganisms with pus cells in $20.4 \%$ of samples, while culture positivity rate was $37.8 \%$. The Gram negative isolates were isolated more frequently than Gram positive isolates i.e., 70\% versus 30\%. Among Gram negative isolates, main isolates were Escherichia coli (E.coli) (74\%), Acinetobacter spp. (11.5\%). Enterobacter spp. (5.8\%), Pseudomonas aeruginosa (5.8\%) and Klebsiella oxytoca (2.9\%). In Gram positive isolates, Staphylococcus aureus (S. aureus), Enterococcus faecalis and Coagulase negative Staphylococcus (CoNS) were 73.3\%, 20\% and 6.7\% respectively. ESBLs were detected among $17.6 \%$ of E. coli and $30.5 \%$ of Klebsiella oxytoca isolates and all were multidrug resistant. Among gram negative isolates the rate of resistance to amoxyclav, ceftazidime, cefotaxime, ceftriaxone, amikacin and ciprofloxacin was $80 \%, 93 \%, 50 \%, 70 \%, 60 \%$ and $50 \%$ respectively. All the strains were found to be sensitive to imipenem. For Gram positive isolate resistance to cephalosporins and amikacin varied from $50 \%$ to $100 \%$. All isolates of $S$. aureus were sensitive to clindamycin and vancomycin and $90 \%$ were sensitive to amoxyclav and ciprofloxacin. None of the isolate was found to be resistant to methicillin (MRSA). One isolate (33\%) of Entercoccus faecalis was resistant to vancomycin (VRE).

\section{DISCUSSION}

Ascites can result from a variety of conditions such as chronic liver disease, malignancy, congestive heart failure, nephrotic syndrome, TB and pancreatic diseases. Cirrhosis of liver accounts for $75 \%$ of all patients of ascites followed by malignancy (10-12\%) and congestive heart failure (5\%). ${ }^{7}$ The recommendations of the American Association for the Study of Liver Disease (AASLD) differ from those of the International Ascites Club. The AASLD recommends testing of ascitic fluid for cell count and differential count, but not necessarily culture, for patients undergoing serial outpatient therapeutic paracentesis. ${ }^{8}$ If ascitic fluid infection is suspected i.e., in presence of fever, abdominal pain, unexplained encephalopathy, acidosis, azotemia, hypotension, or hypothermia, bacterial culture of the fluid in aerobic and anaerobic blood culture bottles inoculated at the bedside should be performed. ${ }^{8}$

The ascitic fluid PMN leukocyte concentration is the best index for the rapid presumptive diagnosis of SBP. Recent advances have improved the evaluation of ascitic fluid. Among them are serum albumin/ascites albumin 
difference, estimation of lactoferrin, PMN counting by automated blood cell counters, using reagent strips and molecular methods i.e., PCR, RFLP and high resolution melting analysis ( HRMA). ${ }^{9-11}$ Familiarity on the part of clinician with ascitic fluid interpretation in various diseases will increase the chances of controlling ascites early. The low concentration of bacteria in ascitic fluid explains the low sensitivity of conventional culture techniques and also explains the low sensitivity of Gram's staining.

In the present study, $20.4 \%$ of the samples showed presence of microorganisms on Gram's staining whereas Runyon et $a l^{12}$ and Sainz et $a l^{13}$ have demonstrated microorganisms in only $6 \%$ cases of SBP. The culture positivity of the ascitic fluid in the current study was $37.8 \%$ which is comparable to other studies. ${ }^{4,14-16}$

Various studies have shown that bedside inoculation of the ascitic fluid into blood culture bottles significantly increases the detection rate of the microorganisms. It also provides quicker identification of the causative organisms. ${ }^{4,14-16}$ Optimal sensitivity is achieved when at least $10 \mathrm{ml}$ of ascitic fluid is inoculated into the blood culture bottles.

In the present study, the low culture positivity rate could be because of less incubation time (single blind subculture after 24 hours) and use of different culture media (BHI) instead of multiple blind subculture and use of Columbia broth. ${ }^{4}$

Bacteria isolated from the ascitic fluid in patients with SBP are usually those of the normal intestinal flora. In the present study, Gram negative isolates were isolated more frequently than Gram positive isolates. Among Gram negative isolates, E.coli was most frequently isolated organism followed by Acinetobacter spp., Enterobacter spp., Pseudomonas aeruginosa and Klebsiella oxytoca. Similar findings has also been reported from other studies. ${ }^{4,16}$

Recent reports have described a change in the bacteriological profile of SBP to Gram positive isolate, especially in patients who were on long-term prophylaxis. ${ }^{17-19}$ Amongst the Gram positive isolates, S. aureus, Enterococcus faecalis and CoNS were 73.3\%, $20 \%$ and $6.7 \%$ respectively.

SBP is only rarely caused by anaerobic organisms or by more than one type of bacteria, so their presence in ascitic fluid should raise suspicion of bacterial peritonitis due to some other cause. It has been suggested that the microbiological causes of SBP and the susceptibility of the causative organisms to antibiotics are changing for several reasons. Since SBP is a serious complication in cirrhotic patients, empirical antibiotic therapy by third generation cephalosporins is usually initiated before the results of the ascitic fluid culture and sensitivity are available. In the present study, among Gram negative isolates, rate of resistance to third generation cephalosporins varies from 50-93\%. However Gram negative isolates were found to be relatively sensitive to amikacin and ciprofloxacin. All the strains were found to be sensitive to imipenem. Other studies have recommended use of meropenem, in severe cases as well as in ESBL producing E.coli causing SBP. $^{20}$

ESBL producing strains have emerged among Enterobacteriaceae such as E. coli and Klebsiella spp., with an estimated prevalence of $25-35 \%$ among hospitalized patients. ${ }^{1721}$ In the present study ESBLs production was seen in $17.6 \%$ of E.coli and $30.5 \%$ of Klebsiella spp. isolates. ${ }^{21}$ All the ESBL producing strains were multidrug resistant.

All the S.aureus isolates were sensitive to clindamycin and vancomycin but only $90 \%$ were sensitive to amoxyclav and ciprofloxacin which is comparable to the study by Ahmad et al. ${ }^{22}$

It is advocated that empiric therapy may be started with imipenem. The drug therapy may be modified later on the basis of subsequent culture and drug susceptibility report. The limitation of the current study was low isolation rate of culture, which could have been increased by multiple subculture and use of better enrichment media.

The current study highlights the bacterial flora responsible for SBP and their susceptibility pattern in northern India. Among Gram negative isolates, E. coli was most common isolate followed by Acinetobacter spp. and among Gram positive isolates, S. aureus was the commonest isolate followed by Enterococcus faecalis. The knowledge of causative organism and their antibiotic resistance profile will help the clinician in choosing appropriate empirical antibiotic therapy in 
patients with ascites. We also recommend that ESBL producing pathogens should be properly identified and their antibiotic resistance pattern be confirmed and communicated to the physicians for appropriate patient management.

\section{Conflict of interest: None.}

\section{REFERENCES}

1. Conn HO, Fessel JM. Spontaneous bacterial peritonitis in cirrhosis: variations on a theme. Medicine 1971;50(3):161-97.

2. Fernandez J, Bauer TM, Navasa M, Rodes J. Diagnosis, treatment, and prevention of spontaneous bacterial peritonitis. Bailliere's Best Pract Res Clin Gastroenterol 2000;14: 975-90.

3. Thomas V. Routine analysis of cirrhotic ascites for evidence of infection-not worth the effort? Indian J Gastroenterol 2011;30(5):201-3.

4. Siersema PD, de Marie S, van Zeijl JH, Bac DJ, Wilson JH. Blood culture bottles are superior to lysis-centrifugation tubes for bacteriological diagnosis of spontaneous bacterial peritonitis. J Clin Microbiol 1992;30:667-9.

5. Clinical and Laboratory Standards Institute. Performance Standards for Antimicrobial Susceptibility Testing; TwentySecond Informational Supplement. CLSI document M100S22.Clinical and Laboratory Standards Institute, Wayne, Pennsylvania USA; 2012.

6. Kader AA, Angamuthu KK, Kamath KA, Zamon MN. Modified double-disc test for detection of extended spectrum $\beta$ lactamases in Escherichia coli and Klebsiella pneumoniae. Br J Biomed Sci. 2006;63(2):51-4

7. Bac DJ, Siersema PD, Wilson JH. Paracentesis. The importance of optimal ascitic fluid analysis. Neth J Med. 1993;43:147-55.

8. Runyon BA. Management of adult patients with ascites caused by cirrhosis. Hepatology 2004;39(3):841-56.

9. Rogers GB, Russel LE, Preston PG, Marsh P, Collin JE, Saunders $\mathrm{J}$, et al. Characterisation of bacteria in ascitesreporting the potential of culture - independent, molecular analysis. Eur J Clin Microbiol Infect Dis 2010;29(5):533-41.

10. Hardick J, Won H, Jeng K, Hsieh Y, Gaydos CA, Richard E, et al. Identification of bacterial pathogens in ascitic fluids from patients with suspected spontaneous bacterial peritonitis by use of Broad range PCR (16S PCR) coupled with High - Resolution Melt Analysis. J Clin Microbiol 2012;50:2428-32.
11. Sarwar S, Alam A, Izhar M, Khan AA Butt AK, Shafqat F, et al. Bed side diagnosis of spontaneous bacterial peritonitis using reagent strips. J Coll Physiciac Surg Pak 2005;15(7):418-21.

12. Runyon BA, Canawati HN, Akriviadis EA. Optimization of ascitic fluid culture technique. Gastroenterology 1988; 95 (5) : 1351-5.

13. Sainz S, Soriano G, Coll P, Teixidó M, Alonso C, Such J, et al. Spontaneous bacterial peritonitis: a comparative study of 2 methods of ascitic fluid culture. Rev Esp Enferm Dig. 1990; 78(2):76-8.

14. Runyon BA, Umland ET, Merlin T. Inoculation of blood culture bottles with ascitic fluid: improved detection of spontaneous bacterial peritonitis. Arch Intern Med 1987;147(1):73-5.

15. Chareonhirunyinggyos S, Dhiraputra C, Leelarasamee A. Bacterial isolation with onsite inoculation of ascitis fluid into hemoculture bottle in spontaneous bacterial peritonitis. J Med Assoc Thai 2004;87:486-90.

16. Rajput MR, Zuberi BF, Shaikh WM, Solangi GA, Shaikh SM, Shaikh GM. Frequency, microbial spectrum, clinical and biochemical features of spontaneous bacterial peritonitis and its variants. J Coll Physicians Surg Pak 1999;9(8):347-50.

17. Fam N, Ghali A, Sayed A, Klena JD. Changes in etiologic and antibiotic resistance profiles of bacteria causing spontaneous peritonitis in Egyptian patients with liver cirrhosis. Egypt J Med Microbiol 2008;17:199-208.

18. Park MK, Lee JH, Byun YH, Lee HIe, Gwak GY, Choi MS, et al. Changes in the profiles of causative agents and antibiotic resistance rate for spontaneous bacterial peritonitis: an analysis of cultured microorganisms in recent 12 years. Korean J Hepatol 2007;13(3):370-7.

19. Cholonqitas E, Paptheochoridis GV, Lahanas A, Xan-thnki A, Kontou-Kastellanou, Archimendritis AJ. Increasing frequency of Gram positive bacteria in spontaneous bacterial peritonitis. Liver Int 2005;25(1):57-61.

20. Paterson DL. Recommendation for treatment of severe infections caused by Enterobacteriaceae producing extended spectrum beta-lactamases (ESBLs). Clin Microbiol Infect 2000;6(9):460-3.

21. Fam N, Diab M, Helmi H, El-Defrawy I. Phenotypic detection of metallo $\beta$-lactamases and extended-spectrum $\beta$ - lactamases among Gram-negative bacterial clinical isolates. Egypt J Med Microbial 2006;15:719-30.

22. Ahmed M, Ali AA, Mumtaz M, Iqbal J, Mughal AA. Spontaneous bacterial peritonitis; Microbiological analysis of ascitic fluid in patients with complicated liver cirrhosis Professional Med J 2011;18(4):557-61 